\title{
Pyridoxamine improves diabetes-evoked delayed bone repair in mice
}

\author{
Shuhei Ugaji ${ }^{1,2 \#}$, Kumi Kimura ${ }^{2 \#}$, Yasuhiko Yamamoto ${ }^{2 *}$, Seiichi Munesue ${ }^{2}$, Ai Harashima ${ }^{2}$, Takao Aikawa ${ }^{1}$, Yasuhisa Yoshida ${ }^{1}$, Tomo \\ Hamada $^{1}$, Toshifumi Hikichi ${ }^{1}$, Hidenori Matsubara ${ }^{1 *}$ and Hiroyuki Tsuchiya ${ }^{1}$ \\ ${ }^{1}$ Department of Orthopaedic Surgery, Kanazawa University Graduate School of Medical Sciences, Kanazawa 920-8641, Japan \\ ${ }^{2}$ Department of Biochemistry and Molecular Vascular Biology, Kanazawa University Graduate School of Medical Sciences, Kanazawa 920-8640, Japan \\ \#Equally contributed
}

\begin{abstract}
Diabetes mellitus is associated with a risk of delayed bone healing and pseudoarthrosis. The production of highly reactive intermediate derivatives of glucose metabolism, including methylglyoxal (MGO), and subsequent acceleration of the glycation reaction under chronic sustained hyperglycemia are implicated in diabetic complications. Pyridoxamine (PM), a natural form of vitamin B6, has been reported to inhibit glycation and proposed to be effective in restraining the complications of diabetes. In the present study, we examined the effectiveness of PM treatment in disturbed bone healing in diabetic mice and MGO-induced osteoblast dysfunction. Multiple injections of low-dose streptozotocin (STZ) $(50 \mathrm{mg} / \mathrm{kg}$ body weight for 5 days) were injected to induce diabetes in mice. After confirming overt hyperglycemia, a drill-hole injury $(5.0 \mathrm{~mm})$ was introduced into the mouse femur and bone defect healing was evaluated with or without PM treatment. Although the diabetic status was not affected by PM treatment, computed tomography scanning images revealed that delayed bone healing was significantly improved in the early phase on days 3 and 10 after injury; the result is also supported by a histological evaluation. In vitro cell-based experiments showed that the osteoblastic differentiation in MC3T3 cells was significantly inhibited by MGO exposure in a dose-dependent manner, but the impairment was recovered by PM treatment. In conclusion, PM, a water-soluble vitamin, could be a useful candidate for recovery against bone injury induced by MGO toxicity in diabetes.
\end{abstract}

\section{Introduction}

Diabetes mellitus is a metabolic disorder, and its prevalence is increasing worldwide. It is a major cause of cardiovascular disease, stroke, chronic kidney disease, neuropathy, blindness, and cancer, which are closely associated with morbidity and mortality in patients with diabetes. Diabetes has also been recognized to affect bone health in terms of bone strength, turnover, mineral density, and structure. Delayed bone healing is recognized as an additional complication of longstanding diabetes, which is associated with a high risk of delayed union, non-union, or pseudoarthrosis [1-3]. Prevention and improvement of the disabling bone complications in diabetes have attracted more attention.

Considering the underground mechanisms of hyperglycemia, glycative stress and oxidative stress are known to be associated with the development of diabetic complications including delayed bone healing due to reduced osteoblast differentiation, increased osteoclast activity, and induction of apoptosis in chondrocytes and osteoblasts [4-7]. Glycative stress is an overwhelming and unfavorable glycation state that has been established as a pathogenic factor. Glycation is a non-enzymatic reaction that ultimately results in the formation of advanced glycation end-products (AGEs) in biological macromolecules. The characteristic increase in glycative stress under high glucose conditions in vivo leads to diabetic complications due to accumulation of AGEs from exogenous and endogenous sources and the production of dicarbonyls, which are reactive compounds and major AGE precursors. The importance of glycative stress is proved by the fact that hemoglobin A1c (HbAlc), a compound with an Amadori rearrangement produced during early glycation reaction, is closely correlated with the development of diabetic complications, and is clinically used as a surrogate marker of an average blood glucose level over approximately one month [8-10]. AGEs and dicarbonyls are recognized as pro-inflammatory and pro-oxidant mediators that lead to various biological responses, predominantly by activating the receptor for AGEs.

We have previously reported that glycative stress and accumulation of AGEs elicit delayed bone healing in mice [11]. The most reactive dicarbonyl, methylglyoxal (MGO), inhibits osteoblast differentiation [11]. Thus, the production of MGO should be inhibited to elucidate its beneficial role against bone problems in diabetes. Currently, pyridoxamine $(\mathrm{PM})$ is receiving considerable attention as an AGE inhibitor and an investigational drug for the treatment of diabetic complications. PM is one of the three natural forms of vitamin B6, together with pyridoxal (PL) and pyridoxine (PN), and has been identified as an anti-glycating agent that act via trapping of a-dicarbonyl compounds [12-21]. Previous studies have shown that PM inhibits the formation of AGEs and retards the development of

${ }^{\star}$ Correspondence to: Yasuhiko Yamamoto, Department of Biochemistry and Molecular Vascular Biology, Kanazawa University Graduate School of Medical Sciences, Kanazawa 920-8640, Japan, E-mail: yasuyama@med.kanazawa-u.ac.jp

Hidenori Matsubara, Department of Orthopaedic Surgery, Kanazawa University Graduate School of Medical Sciences, Kanazawa 920-8641, Japan, E-mail: ortho331@ staff.kanazawa-u.ac.jp

Key words: metylglyoxal, glycation, bone injury repair, pyridoxamine

Received: February 23, 2021; Accepted: March 17, 2021; Published: March 19, 2021 
diabetic nephropathy and retinopathy in animal models of diabetes [22]. In the present study, we aimed to examine the effectiveness of PM treatment in improving bone repair ability in vivo using hyperglycemic diabetic mice, along with restoration of MGO-induced derangement of osteoblast differentiation in vitro.

\section{Materials and methods}

\section{Cell culture}

Mouse MC3T3 cells (Riken Cell Bank, Tsukuba, Japan) were maintained in $\alpha$ - minimum essential medium $(5.5 \mathrm{mmol} / \mathrm{L}$ glucose; Wako Pure Chemical Industries, Osaka, Japan), supplemented with $10 \%$ fetal bovine serum, $100 \mathrm{U} / \mathrm{mL}$ penicillin, and $100 \mu \mathrm{g} / \mathrm{mL}$ streptomycin in a $5 \% \mathrm{CO}_{2}$ atmosphere at $37^{\circ} \mathrm{C}$. To induce osteoblastic differentiation in MC3T3 cells, $10 \mathrm{mM} \beta$-glycerophosphate, $0.1 \mu \mathrm{M}$ dexamethasone, and $50 \mu \mathrm{M}$ ascorbic acid were added to the cell culture medium. For the assays, $0.5-2.0 \mathrm{mM}$ MGO (Sigma Aldrich) and $0.3-3.0 \mathrm{mM}$ pyridoxamine-dihydrochloride-monohydrate (4-aminomethyl-3hydroxy-2-methyl-5-oxymethylpyridihydrochloride, Tokyo chemical industry, Japan) was added to the differentiation medium, and the medium was changed every other day.

\section{Alkaline phosphatase (ALP) activity assay}

ALP activity in MC3T3 cells was determined 7 days after the induction of osteoblastic differentiation, using a TRACP \& ALP Assay kit (Takara Bio, Inc., Otsu, Japan), according to the manufacturer's protocol.

\section{Experimental animals and induction of diabetes}

Male C57BL/6J mice (weight, approximately $21 \mathrm{~g}$ ) at 6 weeks of age were purchased from Charles River Japan, Inc. (Yokohama, Japan). They were acclimatized for 1 week prior to the start of the experiment. For diabetes induction, streptozotocin (STZ; $50 \mathrm{mg} / \mathrm{kg}$ body weight) was intraperitoneally injected daily for 5 days according to the Animal Models of Diabetic Complications Consortium (AMDCC) protocols. In contrast, sodium citrate buffer was injected into mice of non-diabetic control group. PM was administered to the animals in drinking water $(1.0 \mathrm{~g} / \mathrm{L})$. Mice were divided into four experimental groups: non-diabetic control $(\mathrm{CNT})$ groups with or without PM treatment [CNT+PM (n = $10)$ or CNT $(n=10)$, respectively] and diabetic groups with or without PM treatment [diabetic mice $(D M)+P M(n=10)$ or DM $(n=10)$, respectively]. After 3 weeks of STZ or sodium citrate buffer injection, non-fasting blood glucose levels were monitored with a glucometer (Glutest Ace, Sanwa Kagaku, Japan) using whole blood obtained from the tail vein. Diabetes was identified by a blood glucose level of $>300 \mathrm{mg} / \mathrm{dL}$. HbAlc, an early glycation marker, was measured in the tail vein blood using DCA Vantage analyzer (Siemens Healthineers, Erlangen, Germany). Mice were maintained under standard cage conditions $\left(24{ }^{\circ} \mathrm{C} ; 12 / 12 \mathrm{~h}\right.$ light/dark cycle) with sawdust bedding, and access to food (mouse standard chow diet) and water ad libitum. All animal experiments were approved by the Committee on Animal Experimentation of Kanazawa University and performed in accordance with the Fundamental Guidelines for Proper Conduct of Animal Experiment and Related Activities in Academic Research Institutions under the jurisdiction of the Ministry of Education, Culture, Sports, Science, and Technology of Japan.

\section{Induction of drill hole injury in the femur}

A straight and longitudinal skin incision $(5.0 \mathrm{~mm})$ was made in the mouse distal femur under anesthesia using combination anesthetic containing midazolam $(4.0 \mathrm{mg} / \mathrm{kg})$, medetomidine $(0.3 \mathrm{mg} / \mathrm{kg})$, and butorphanol $(5.0 \mathrm{mg} / \mathrm{kg})$. The periosteal membrane was then stripped away, followed by splitting of medial great muscle to expose the femur bone surface. A drill hole injury was introduced by inserting a drill bit ( $0.9 \mathrm{~mm}$ in diameter) at the medial portion of the diaphysis of the left femur, $5 \mathrm{~mm}$ above the knee joint, as described previously [11]. The hole was drilled through the collateral cortical bone and bone marrow. During surgery, the body temperature was maintained at $37^{\circ} \mathrm{C}$ using a heating pad.

\section{Computed tomography (CT) scanning}

Whole femur bones were scanned under anesthetic condition using an X-ray CT system (Latheta LCT 200; Hitachi Aloka Medical, Tokyo, Japan) at $0,3,7,10$, and 14 days after the drill hole injury. The healing process in the bone defect lesions was evaluated using a suitable analysis software (AzeWin; AZE, Ltd., Tokyo, Japan). Images were processed in a multiplanar reconstruction, according to oblique coronal planes, maintaining the working axes parallel to the center line of the bone defect. CT values at the area of the bone defect were calculated at every phase, as described previously [11].

\section{Histopathological examination}

The left femur was extracted and fixed in $10 \%$ buffered formaldehyde solution, 7 days after the drill hole injury. After $12 \mathrm{~h}$ of fixation, soft tissues were cleaned, and specimens were decalcified in formic acid sodium nitrate solution as described previously. The specimens with a drill hole were sectioned and embedded in paraffin. Mid-sagittal serial sections ( 5 to $7 \mu \mathrm{m}$ thick) were prepared and stained with hematoxylin and eosin (H\&E). Histological sections were examined using light microscopy at a magnification of $100 \times$.

\section{Statistical analysis}

Data are presented as the mean \pm standard error of the mean. The differences in the measured variables among the four groups were analyzed using non-repeated one-way analysis of variance (ANOVA). Post hoc multiple comparison using Tukey's method was performed to assess differences between the groups. Differences with a p value $<0.05$ were considered significant. Statistical analysis was performed using the SPSS software (IBM SPSS Statistics Version 22, IBM, Armonk, NY, USA).

\section{Results}

\section{Effects of PM treatment on bone defect repair in STZ-induced diabetic mice}

To examine the effectiveness of PM treatment on bone defect repair in diabetes, DM and CNT were used with or without PM treatment (1 $\mathrm{g} / \mathrm{L}$ ), which was given to mice in the drinking water. We first evaluated hyperglycemic conditions related to diabetes. As a result, overt diabetic status was observed in STZ-induced DM compared to CNT: body weight ( $22 \pm 1.4 v s .27 \pm 1.7 \mathrm{~g})$, non-fasting blood glucose levels $(531 \pm$ 94.8 vs. $132 \pm 10.6 \mathrm{mg} / \mathrm{dL})$, and $\mathrm{HbAlc}(7.9 \% \pm 0.99 \%$ vs. $3.7 \% \pm 0.28 \%)$ $(p<0.05)$ at 3 weeks after multiple low-dose STZ injections $(50 \mathrm{mg} / \mathrm{kg}$ body weight, daily for 5 days) (Table 1). Polydipsia was evident in DM group, compared to CNT group $(9.3 \pm 0.86$ vs. $3.3 \pm 0.22 \mathrm{~g} /$ day; DM vs. CNT) (Table 1). PM treatment did not affect the diabetic status of DM group (body weight, $22 \pm 1.4 \mathrm{~g} v$ s. $22 \pm 1.7 \mathrm{~g}$; blood glucose, $531 \pm 94.8$ vs. $539 \pm 63.0 \mathrm{mg} / \mathrm{dL}$; HbAlc, $7.9 \% \pm 0.99 \%$ vs. $7.7 \% \pm 0.36 \%$; water intake, $9.3 \pm 0.86$ vs. $6.6 \pm 3.82 \mathrm{~g} /$ day; $\mathrm{DM} v s$. DM+PM, respectively) (Table 1). In addition, PM did not change any indices of CNT group (Table 1). 
Further, we investigated bone defect repair of drill hole defects in the mouse femur, which was evaluated using CT scanning. CT images revealed delayed bone repair in the drill hole lesions in DM group compared to $\mathrm{CNT}$ and $\mathrm{DM}+\mathrm{PM}$ groups (Figure 1A). Quantitative evaluations demonstrated that the CT values of the bone defect portion in DM group were significantly lower than those in CNT group, even at 3 days after the injury (Figure 1B). We observed significantly impaired bone healing in DM group compared to the CNT group, during all observation periods $(3,7,10$, and 14 days) (Figure 1B). However, PM treatment significantly improved bone healing in DM group at 3, 7, and 10 days after the drill hole injury (Figure 1B). Moreover, a negative correlation between individual $\mathrm{HbAlc}$ and $\mathrm{CT}$ values at 7 days after the injury, was obtained for DM group (Figure 2), indicating that elevated level of glycated $\mathrm{HbAlc}$ could be associated with delayed bone healing and low CT values. However, PM treatment (DM+PM) group did not show a negative correlation (Figure 2).

Furthermore, histological findings also showed that the bone hole defect was not repaired in DM group, compared to other groups in the restoration stage at 10 days after the injury (Figure 3). PM-treated $(\mathrm{DM}+\mathrm{PM})$ group showed recovered bone hole filled with new bone tissues, as seen in the CNT and CNT+PM groups (Figure 3).

\section{Effects of PM treatment on MGO-induced deterioration of osteoblastic differentiation of MC3T3 cells in vitro}

In vitro cell-based assays demonstrated that exposure to MGO inhibited osteoblastic differentiation in MC3T3 cells, as indicated by
ALP activity, in a dose-dependent manner (Figure 4). We performed in vitro cell-based assays to evaluate the effectiveness of PM treatment on osteoblastic differentiation and observed significant improvement in MGO-induced deterioration of osteoblastic differentiation in MC3T3 cells (Figure 4).

\section{Discussion}

The present study is the first to demonstrate the effectiveness of PM treatment in restoration of delayed healing of bone defect in diabetic mice with a femur drill-hole injury $(1.0 \mathrm{~mm})$ model (Figures 1 and 3 ). $\mathrm{CT}$ and histological evaluations revealed that the ossification recovery rate of the bone defect lesion in DM+PM group was almost similar to that in CNT group (Figures 1 and 2), although PM treatment was found to have no significant effects on lowering blood glucose or HbAlc levels in STZ-induced diabetic mice (Table 1). Individual data analyses demonstrated that DM mice with higher HbAlc and average blood glucose levels showed lower CT values in the drilled bone defect portion at 7 days after surgery (Figure 2), compared to (DM+PM) group with no negative correlation between $\mathrm{HbAlc}$ and CT values. The dosage of PM (1 g/L drinking water; approximately $200 \mathrm{mg} / \mathrm{kg} /$ day) used in this study was the same as in our previous report, which proved it to be a safe dose $(0.42 \mu \mathrm{M})$ that attained serum concentrations of PM within a less toxic range in vivo, and its preclinical efficacy has been proven in diabetic kidney disease in KK-Ay/Ta and STZ-induced diabetic rats and mice $[17,14]$. Notably, PM treatment did not accelerate the ossification recovery in $\mathrm{CNT}+\mathrm{PM}$ group (Figures 1 and 3), suggesting that PM

Table 1. Diabetic status of mice.

\begin{tabular}{|c|c|c|c|c|}
\hline Group & BW (g) & BG (mg/dl) & HbA1c (NGSP, \%) & Amount of drinking water (g/day) \\
\hline CNT & $27 \pm 1.7^{*}$ & $132 \pm 10.6^{*}$ & $3.7 \pm 0.28^{*}$ \\
\hline DM & $22 \pm 1.4$ & $531 \pm 94.8$ & $7.9 \pm 0.99$ \\
\hline DM+PM & $22 \pm 0.7$ & $539 \pm 63.0$ & $7.7 \pm 0.36$ \\
\hline CNT+PM & $28 \pm 1.7^{*}$ & $163 \pm 75.5^{*}$ & $6.3 \pm 0.86$ \\
\hline
\end{tabular}

${ }^{*} p<0.05$ vs. DM or DM+PM; the data are expressed as mean \pm standard error of the mean. BW, body weight; BG, blood glucose; NGSP, National Glycohemoglobin Standardization Program; CNT, control; DM, diabetes mellitus; PM, pyridoxamine.

A

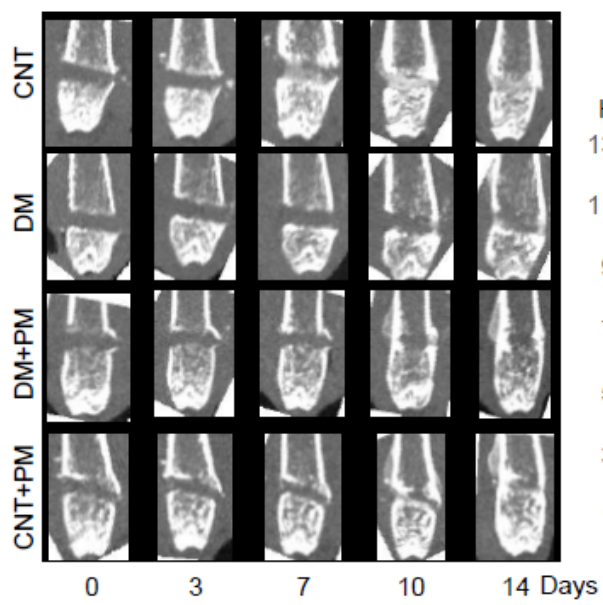

B

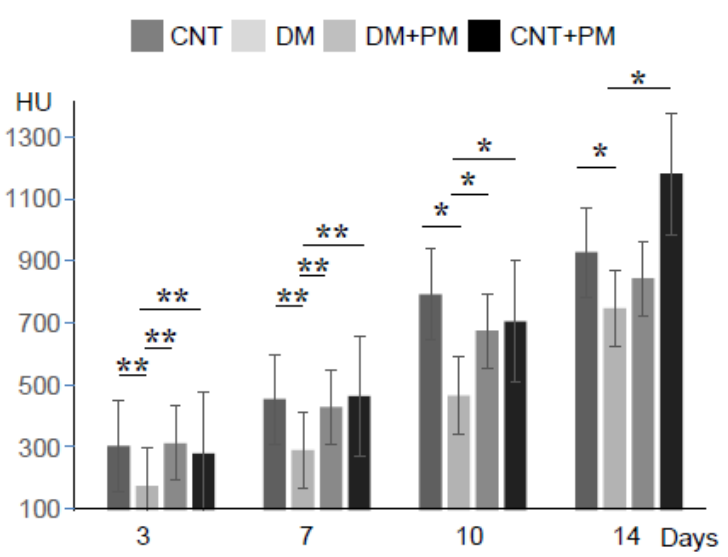

Figure 1. Evaluation of bone defect repair by CT scanning. (A) CT images at 0,3, 7, 10, and 14 days after bone injury in the left femur. (B) Quantitative evaluation of the CT imaging. A vertical axis indicates CT values of the bone defect lesion. CNT, non-diabetic control groups without pyridoxamine (PM) treatment; CNT+PM, non-diabetic control groups with PM treatment; DM, diabetic groups without PM treatment; DM+PM, diabetic groups with PM treatment; HU, hounsfield unit. Data are presented as the mean $\pm \mathrm{SEM} ; \mathrm{n}=10$ per group. *, $p<$ $0.05 ; * *, p<0.01$ 


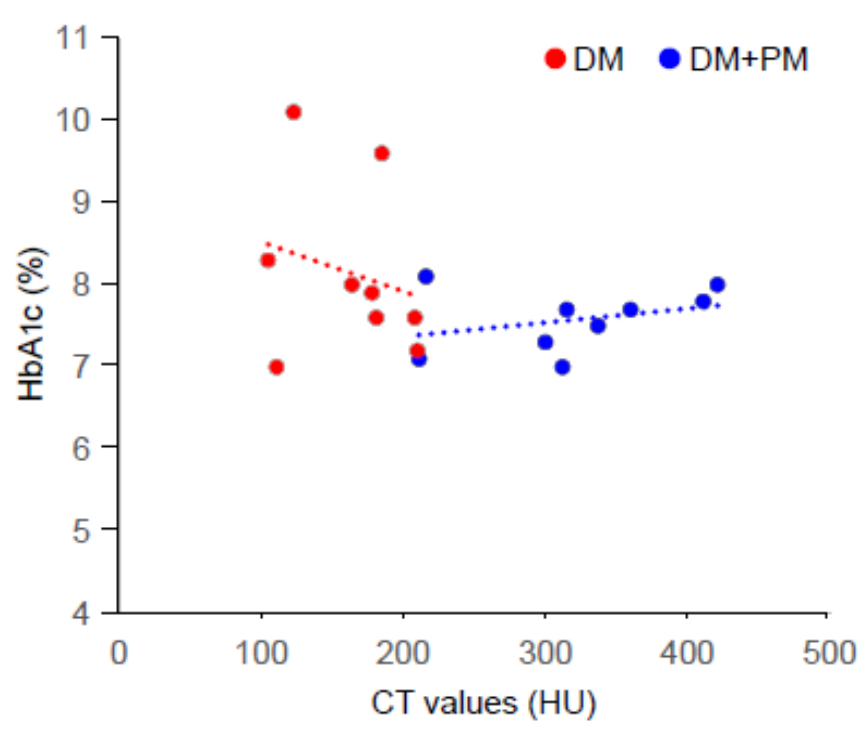

Figure 2. Correlation diagrams between HbAlc and CT values at 3 days after the bone injury. DM, diabetic groups without PM treatment; DM+PM, diabetic groups with PM treatment; $\mathrm{n}$ $=10$ per group

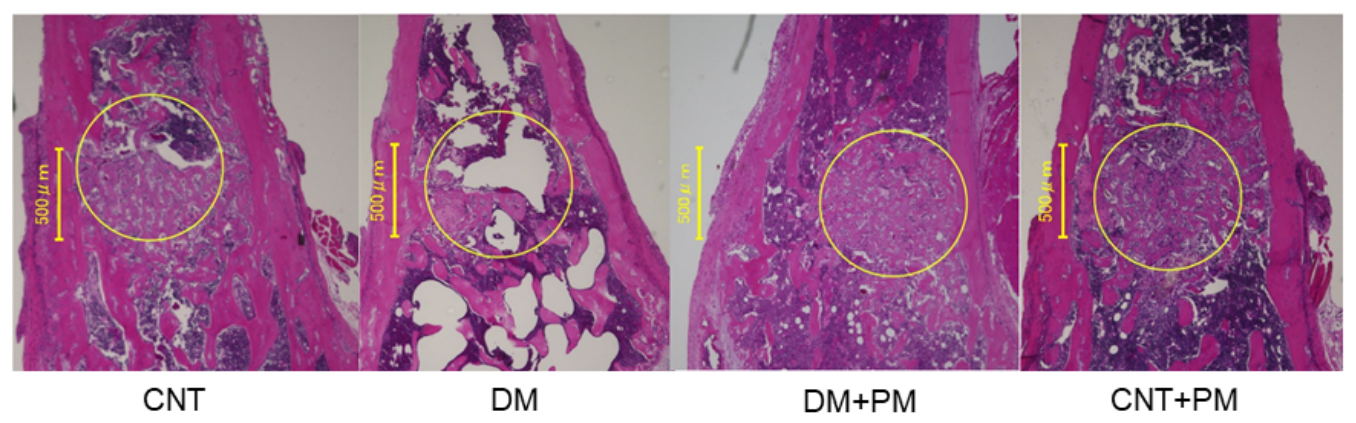

Figure 3. Microscopic findings at day7 after the bone injury. CNT, non-diabetic control groups without PM treatment; CNT+PM, non-diabetic control groups with PM treatment. H\&E stain. Magnification, $\times 10$
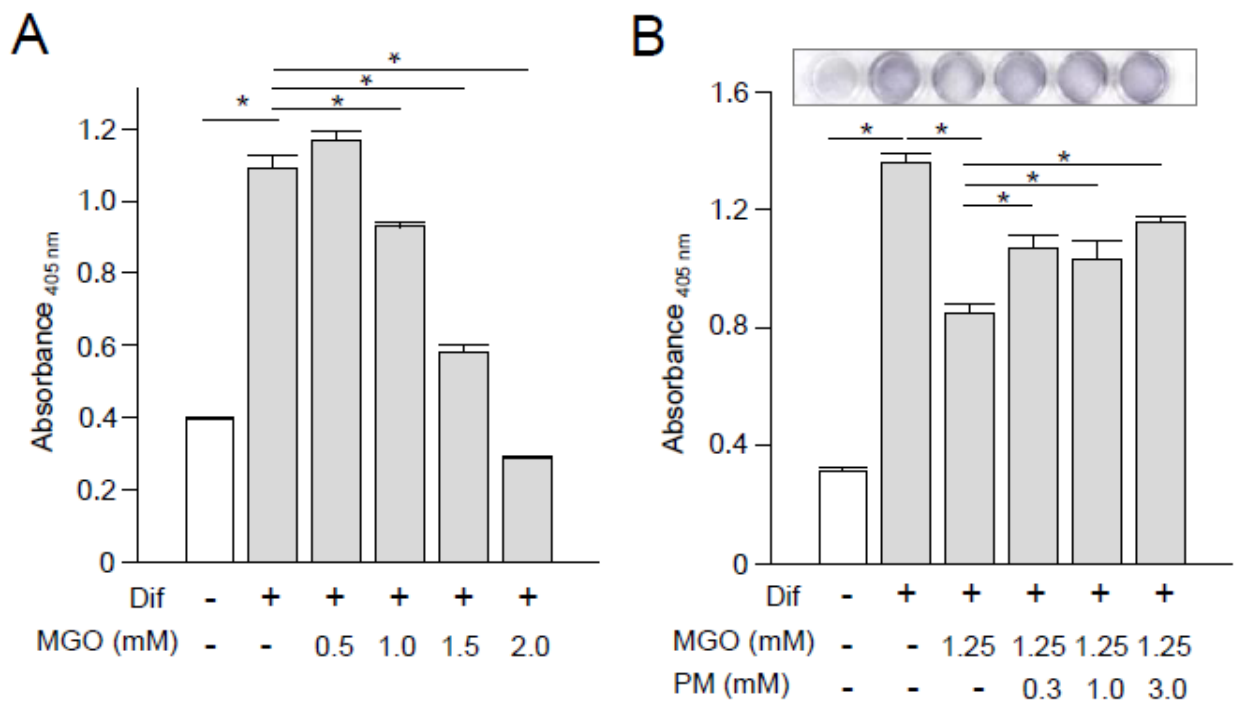

Figure 4. In vitro osteoblastic differentiation of MC3T3 cells. (A and B) Alkaline phosphatase (ALP) activity assay using MC3T3 cells at 7 days after the induction of osteoblastic differentiation. Dif, differentiation media; MGO, methylglyoxal; PM, pyridoxamine. Data are presented as the mean \pm SEM; $\mathrm{n}=6$ per group. ${ }^{*}, p<0.05$ 
could affect only diabetes- and hyperglycemia-derived metabolic alterations in the bone repair tissues.

In vitro assay of MC3T3 cells revealed that the addition of MGO into cell culture medium decreased ALP activity, which is the most frequently used marker for osteoblast differentiation and functions (Figure 4). However, PM treatment significantly improved MG-induced deterioration of osteoblastic differentiation in MC3T3 cells (Figure 4). In this assay, we used MGO at concentrations of 0.5 to $2.0 \mathrm{mM}$ equivalent to those in our previous study [11]. The concentration of MGO in vivo remains controversial, and the data vary among multiple assay methods. The actual intracellular concentration of MGO has been argued in living organs and cells. In addition, the concentrations of PM used for in vitro cell-based experiments are still under discussion along with MGO doses. Pyridoxine, a form of vitamin B6, has been reported to cause cell death at $1 \mu \mathrm{M}$ [23]. In contrast, pyridoxal and PM are known to be nontoxic. No or minimal adverse events of PM have been observed in previous clinical studies.

DM can cause many complications [24]. The mechanism of diabetes-associated bone problems has been assumed that bone marrow-derived mesenchymal stem cells would not be recruited to the injured site in diabetic patients [25], and therefore, diabetes has been related to osteoblast suppression and osteoclast-promoting actions [24]. Our previous study demonstrated that glycation stress, including MGO, could impair osteoblastic differentiation and delay bone injury repair in diabetes [11]. In this study, PM administration rescued bone injury repair in mice with diabetes. Thus, we propose that inhibition of MGO production could be a potentially useful strategy against bone problems in diabetes and PM could be a powerful candidate for MGO detoxification.

PM, a derivative of vitamin B6, is reported to have many biological effects: (1) inhibition of AGE formation by trapping dicarbonyl intermediates, including MGO, during the glycation reaction, (2) scavenging of toxic carbonyl products of glucose and lipid degradation, and (3) scavenging of reactive oxygen species (ROS) [12]. Oral PM treatment is reported to have benefits related to creatinine clearance and level of urinary transforming growth factor- $\beta 1$ in patients with type 1 and type 2 diabetic nephropathy in a phase II clinical study [18]. In animal studies, PM attenuated glycated serum protein levels and intervertebral disc degeneration process in Zucker diabetic SpragueDawley (ZDSD) rats [26]. A recent study demonstrated the inhibitory effects of short- and long-term treatment of PM on the adhesive function of neutrophils and platelets in the microvessels of sickle cell disease mice [27], suggesting a novel biological action of PM.

In conclusion, our findings suggest that PM treatment could be useful in reducing glycative stress and improving bone union delay associated with diabetes. This is the first report on the beneficial effects of PM in bone injury and provides a basis for the treatment of pseudoarthrosis and delayed bone healing/fusion in patients with diabetes.

\section{Acknowledgements}

We thank Y. Niimura for technical assistance. The document was edited by Editage, a division of Cactus Communications.

\section{Funding}

We acknowledge the financial support from the Kanazawa University SAKIGAKE project 2018, Takeda Science Foundation, and Grants-in-Aid for Scientific Research (16K10896, 18K07551,
20K07323, 19K09593, and 18K06889) and the Program for Fostering Globally Talented Researchers from the Japan Society for Promotion of Sciences.

\section{Declaration of competing interest}

The authors declare no conflicts of interest to report.

\section{References}

1. Retzepi M, Donos N (2010) The effect of diabetes mellitus on osseous healing. Clin Oral Implants Res 21: 673-681. [Crossref]

2. Loder RT (1988) The influence of diabetes mellitus on the healing of closed fractures. Clin Orthop Relat Res 232: 210-216. [Crossref]

3. Folk JW, Starr AJ, Early JS (1999) Early wound complications of operative treatment of calcaneus fractures: Analysis of 190 fractures. JOrthop Trauma 13: 369-372. [Crossref]

4. Kayal RA, Siqueira M, Alblowi J, McLean J, Krothapalli N, et al. (2010) TNF alpha mediates diabetes enhanced chondrocyte apoptosis during fracture healing and stimulates chondrocyte apoptosis through FOXO1. J Bone Miner Res 25: 1604-1615.

5. Botushanov NP, Orbetzova MM (2009) Bone mineral density and fracture risk in patients with type 1 and type 2 diabetes mellitus. Folia Med (Plovdiv) 51: 12-17. [Crossref]

6. Stolzing A, Sellers D, Llewelyn O, Scutt A (2010) Diabetes induced changes in rat mesenchymal stem cells. Cells Tissues Organs 191: 453-465.

7. Sheweita SA, Khoshhal KI (2007) Calcium metabolism and oxidative stress in bone fractures: Role of antioxidants. Curr Drug Metab 8: 519-525. [Crossref]

8. Goldfine AB, Fonseca V, Jablonski KA, Pyle L, Staten MA, et al. (2010) TINSAL-T2D (Targeting Inflammation Using Salsalate in Type 2 Diabetes) Study Team: The effects of salsalate on glycemic control in patients with type 2 diabetes: a randomized trial. Ann Intern Med 152: 346-357.

9. Goldfine AB, Fonseca V, Jablonski KA, Chen YD, Tipton L, et al. (2013) Targeting Inflammation Using Salsalate in Type 2 Diabetes Study Team: Salicylate (salsalate) in patients with type 2 diabetes: a randomized trial. Ann Intern Med 159: 1-12. [Crossref]

10. Barzilay JI, Jablonski KA, Fonseca V, Shoelson SE, Goldfine AB, et al. (2014) TINSAL-T2D Research Consortium: The impact of salsalate treatment on serum levels of advanced glycation end products in type 2 diabetes. Diabetes Care 37: 1083-1091.

11. Aikawa T, Matsubara H, Ugaji S, Shirakawa J, Nagai R, et al. (2017) Contribution of methylglyoxal to delayed healing of bone injury in diabetes. Mol Med Rep 16: 403-409.

12. Voziyan PA, Hudson BG (2005) Pyridoxamine as a multifunctional pharmaceutical: Targeting pathogenic glycation and oxidative damage. Cell Mol Life Sci 62: 1671-1681. [Crossref]

13. Degenhardt TP, Alderson NL, Arrington DD, Beattie RJ, Basgen JM, et al. (2002) Pyridoxamine inhibits early renal disease and dyslipidemia in the streptozotocindiabetic rat. Kidney Int 61: 939-950. [Crossref]

14. Zhu P, Lin H, Sun C, Lin F, Yu H, et al. (2012) Synergistic effects of telmisartan and pyridoxamine on early renal damage in spontaneously hypertensive rats. Mol Med Rep 5: 655-662.

15. Waanders F, van den Berg E, Nagai R, van Veen I, Navis G, et al. (2008) Renoprotective effects of the AGE inhibitor pyridoxamine in experimental chronic allograft nephropathy in rats. Nephrol Dial Transplant 23: 518-524. [Crossref]

16. Alderson NL, Chachich ME, Youssef NN, Beattie RJ, Nachtigal M, et al. (2003) The AGE inhibitor pyridoxamine inhibits lipemia and development of renal and vascular disease in Zucker obese rats. Kidney Int 63: 2123-2133.

17. Tanimoto M, Gohda T, Kaneko S, Hagiwara S, Murakoshi M, et al. (2007) Effect of pyridoxamine (K 163), an inhibitor of advanced glycation end products, on type 2 diabetic nephropathy in KK A(y)/Ta mice. Metabolism 56: 160-167. [Crossref]

18. Williams ME, Bolton WK, Khalifah RG, Degenhardt TP, Schotzinger RJ, et al. (2007) Effects of pyridoxamine in combined phase 2 studies of patients with type 1 and type 2 diabetes and overt nephropathy. Am J Nephrol 27: 605-614. [Crossref]

19. Lewis EJ, Greene T, Spitalewiz S, Blumenthal S, Berl T, et al. (2012) Collaborative Study Group: Pyridorin in type 2 diabetic nephropathy. J Am Soc Nephrol 23: 131-136. [Crossref]

20. Garg S, Syngle A, Vohra K (2013) Efficacy and tolerability of advanced glycation end products inhibitor in osteoarthritis: A randomized, double blind, placebo controlled study. Clin J Pain 29: 717-724. 
21. Nagaraj RH, Sarkar P, Mally A, Biemel KM, Lederer MO, et al. (2002) Effect of pyridoxamine on chemical modification of proteins by carbonyls in diabetic rats: Characterization of a major product from the reaction of pyridoxamine and methylglyoxal. Arch Biochem Biophys 402: 110-119. [Crossref]

22. Abouzed TK, Munesue S, Harashima A, Masuo Y, Kato Y, et al. (2016) Preventive effect of Salicylate and Pyridoxamine on diabetic nephropathy. J Diabetes Res 2016:1786789.

23. Vrolijk MF, Opperhuizen A, Jansen EHJM, Hageman GJ, Bast A, et al. (2017) The vitamin B6 paradox: supplementation with high concentrations of pyridoxine leads to decreased vitamin B6 function. Toxicol In Vitro 44: 206-212. [Crossref]
24. Simpson CM, Calori GM, Giannoudis PV (2010) Diabetes and fracture healing: the skeletal effects of diabetic drugs. Expert Opin Drug Saf 11: 215-220.

25. Shin L, Peterson DA (2012) Impaired therapeutic capacity of autologous stem cells in a model of type 2 diabetes. J Dent Res 87: 1056-1069. [Crossref]

26. Glaeser JD, Ju D, Tawackoli W, Yang JH, Salehi K, et al. (2020) Advanced Glycation End Product Inhibitor Pyridoxamine Attenuates IVD Degeneration in Type 2 Diabetic Rats. Int J Mol Sci 21: 9709.

27. Li J, Jeong SY, Xiong B, Tseng A, Mahon AB, et al. (2020) Repurposing pyridoxamine for therapeutic intervention of intravascular cell-cell interactions in mouse models of sickle cell disease. Haematologica 105: 2407-2419. [Crossref]

Copyright: @2021 Ugaji S. This is an open-access article distributed under the terms of the Creative Commons Attribution License, which permits unrestricted use, distribution, and reproduction in any medium, provided the original author and source are credited. 